Chirurg 2016 $87: 1077$

DOI 10.1007/s00104-016-0315-x

Online publiziert: 17. Oktober 2016

๑) Springer-Verlag Berlin Heidelberg 2016

CrossMark

\section{F. Weber}

Klinik für Allgemein-, Viszeral- und Transplantationschirurgie, Sektion Endokrine Chirurgie,

Universitätsklinikum Essen, Essen, Deutschland

\section{Originalpublikation}

Regenet N, Carrere N, Boulanger G et al (2016) Is the 2-cm size cutoff relevant for small nonfunctioning pancreatic neuroendocrine tumors: A French multicenter study. Surgery 159(3):901-907

Hintergrund und Fragestellung. Die Einteilung neuroendokriner Tumoren des Pankreas (PNET) erfolgt basierend auf dem Ki67-Proliferationsmarker und der Mitoserate in neuroendokrine Tumore (G1 und G2) oder neuroendokrine Karzinome (NEC; G3). Die moderne Bildgebung erlaubt immer häufiger kleine PNET zu diagnostizieren. Für kleine (bis $2 \mathrm{~cm}$ Größe) PNET besteht jedoch keine klar definierte Handlungsempfehlung. Die vorliegende Multicenterstudie wirft erneut die Frage auf, inwieweit der für die Tumorgröße propagierte $2-\mathrm{cm}$ Grenzwert ein guter prognostischer Marker ist.

Material und Methoden. Es handelt sich um eine retrospektive Datenerhebung (1999-2012) an 7 französischen Universitätskliniken. Alle Patienten mit einem nichtfunktionellen PNET von maximal $2 \mathrm{~cm}$ Größe wurden erfasst. Patienten mit einer genetischen Prädisposition wurden ausgeschlossen. 80 Patienten konnten in der Studie ausgewertet werden. Davon entfielen 14 Patienten auf die nichtoperative Gruppe (NOG) und 66 auf die operative Gruppe (OG). Die Diagnose beruhte bei $56 \%$ der Patienten auf einer Pankreasbiopsie. Ansonsten wurde die Diagnose basierend auf dem Chromogranin A, der Bildgebung oder Rezeptorszintigraphie gestellt. Die mittlere Nachbeobachtungszeit betrug 34 Monate.

Ergebnisse. Die mittlere Tumorgröße lag bei $1,5 \mathrm{~cm}$. Bei $85 \%$ der Patienten konnte ein Ki67 bestimmt werden. Es handelte sich hauptsächlich um G1-PNET ( $n=$ $46)$, gefolgt von G2 $(n=19)$. Bei den 66 Patienten der OG wurde in etwa ein Viertel der Fälle eine Pankreatoduodenektomie durchgeführt. Bei 28 Patienten erfolgte eine Pankreaslinksresektion und bei $35 \%$ eine Parenchymresektion. Die Mortalität der OG lag bei $3 \%$. Die Morbidität betrug $44 \%$ (Grad-C-Pankreasfistelrate von $9 \%$ ).

Insgesamt wurden 15 der 80 PNET aufgrund von Lymphknoten-, Fernmetastasen oder Tumorrezidiv als maligne gewertet. Bei 4 der 15 Patienten lag bei Diagnosestellung eine Fernmetastasierung vor. In der NOG entwickelten 2 der 14 Patienten Metastasen, ohne dass sich die Größe des Primarius geändert hatte.

Das mittlere tumorfreie Überleben der 15 Patienten mit einem malignen PNET war signifikant $(p=0,03)$ kürzer und lag bei 16 Monaten (vs. 30 Monate). Basierend auf der ROC (Receiveroperating-characteristic)-Analyse hatte die Tumorgröße einen signifikanten $(p=0,03)$ Einfluss auf das tumorfreie Überleben mit einer AUC („area under the curve") von 0,75. Demgegenüber lag die AUC für den Prognosefaktor Ki67 bei nur 0,57. Für die Tumorgröße als Prädiktor wurde der optimale Grenzwert (Odds Ratio 10,8) bei $1,7 \mathrm{~cm}$ bestimmt (Sensitivität $92 \%$, 1-Spezifität $35 \%$ ).
Diskussion. Die Studie weist zwar methodische Mängel auf (z. B. inhomogene Gruppen, kurze Nachbeobachtungszeit, mäßiger AUC-Wert) fügt sich aber in die Reihe von Publikationen, die aufzeigen, dass der Ki67 kein akkurater Prädiktor für Malignität bei nichtfunktionellen PNET ist. Des Weiteren zeigen die Daten, dass auch der propagierte 2-cm-Grenzwert nicht hinreichend genau das metastasierte PNET vorhersagen kann. Ob der adaptierte Grenzwert hilfreich ist, muss kritisch bewertet werden.

Fazit. Das niedrige maligne Potenzial kleiner PNET muss gegen das vorhandene Risiko einer Operation abgewogen werden. Der Evidenzgrad der vorhandenen Studien erlaubt letztendlich keine abschließende Handlungsempfehlung und erzwingt die individuelle Therapieabwägung. Die vorliegende Studie bestätigt jedoch den nur eingeschränkten Nutzen von Ki67 und Tumorgröße als sichere prädiktive Marker.

\section{Korrespondenzadresse}

\section{Priv.-Doz. Dr. F. Weber}

Klinik für Allgemein-, Viszeral- und Transplantationschirurgie, Sektion Endokrine Chirurgie, Universitätsklinikum Essen Hufelandstr. 55, 45147 Essen, Deutschland frank.weber@uk-essen.de

Interessenkonflikt. F. Weber gibt an, dass kein Interessenkonflikt besteht. 